\title{
Existence and stability of solutions to non-linear neutral stochastic functional differential equations in the framework of G-Brownian motion
}

\author{
Faiz Faizullah" ${ }^{*}$, M Bux², MA Rana² and Ghaus ur Rahman ${ }^{3}$
}

\author{
"Correspondence: \\ faiz_math@ceme.nust.edu.pk \\ 1 Department of Basic Sciences and \\ Humanities, College of Electrical \\ and Mechanical Engineering, \\ National University of Sciences and \\ Technology (NUST), Islamabad, \\ Pakistan \\ Full list of author information is \\ available at the end of the article
}

\begin{abstract}
In the past decades, quantitative study of different disciplines such as system sciences, physics, ecological sciences, engineering, economics and biological sciences, have been driven by new modeling known as stochastic dynamical systems. This paper aims at studying these important dynamical systems in the framework of G-Brownian motion and G-expectation. It is demonstrated that, under the contractive condition, the weakened linear growth condition and the non-Lipschitz condition, a neutral stochastic functional differential equation in the G-frame has at most one solution. Hölder's inequality, Gronwall's inequality, the Burkholder-Davis-Gundy (in short BDG) inequalities, Bihari's inequality and the Picard approximation scheme are used to establish the uniqueness-and-existence theorem. In addition, the stability in mean square is developed for the above mentioned stochastic dynamical systems in the G-frame.
\end{abstract}

MSC: $60 \mathrm{H} 20 ; 60 \mathrm{H} 10 ; 60 \mathrm{H} 35 ; 62 \mathrm{~L} 2 \mathrm{O}$

Keywords: stability; uniqueness; existence; G-Brownian motion; neutral stochastic functional differential equations

\section{Introduction}

Stochastic differential equations (SDEs) have been used profitably in a variety of fields including population dynamics, engineering, environments, physics and medicine. They are used to describe the transport of cosmic rays in space. For the simulation of the transport of energetic charged particles, SDEs solve several important equations such as Parker's transport equation and the Fokker-Planck equation [1]. SDEs are also utilized in the field of finance as they are optimal to modern finance theory and have been broadly employed to model the behavior of key variables; the variables include the asset returns, asset prices, instantaneous short-term interest rate and their volatility. Biologists use these equations to model the achievement of stochastic changes in reproduction on population processes $[2$, 3]. SDEs can be utilized to describe the percolation of a fluid through absorbent structures and water catchment [4]. There is a huge literature on the applications of SDEs in several disciplines of engineering such as computer engineering [5, 6], random vibrations $[7,8]$,

(c) The Author(s) 2017. This article is distributed under the terms of the Creative Commons Attribution 4.0 International License (http://creativecommons.org/licenses/by/4.0/), which permits unrestricted use, distribution, and reproduction in any medium, provided you give appropriate credit to the original author(s) and the source, provide a link to the Creative Commons license, and indicate if changes were made. 
mechanical engineering [9-11], stability theory [12] and wave processes [13]. These nonlinear equations in the framework of G-Brownian motion were studied by Peng [14, 15], Gao [16] and Faizullah [17]. The study of mild solutions for stochastic evolution equations in the G-framework was given by Gu, Ren and Sakthivel [18]. The $p$ th moment stability of solutions to impulsive SDEs in the framework of G-Brownian motion was established by Ren, Jia and Sakthivel [19]. In the G-framework, stochastic functional differential equations were introduced by Ren, Bi and Sakthivel [20] and then generalized by Faizullah [21, 22]. He also studied the $p$-moment estimates for the solutions to these equations [23, 24]. By virtue of linear growth and Lipschitz conditions, the existence theory for the solutions to neutral stochastic functional differential equations in the G-framework (G-NSFDEs) was given by Faizullah [25]. These equations not only depend on the present and past data but also depend on the rate of change of the past data [26, 27]. Subject to the non-linear growth and non-Lipschitz conditions, we do not know whether these equations admit solutions or not, if the solutions are unique or not and if they admit solutions whether they are mean square stable or not. The present article will fill the mentioned gape. Let $0 \leq t_{0} \leq$ $t \leq T<\infty$. Suppose $\kappa:\left[t_{0}, T\right] \times B C\left(\left[-\tau, t_{0}\right] ; \mathbb{R}^{d}\right) \rightarrow \mathbb{R}^{d}, \lambda:\left[t_{0}, T\right] \times B C\left(\left[-\tau, t_{0}\right] ; \mathbb{R}^{d}\right) \rightarrow \mathbb{R}^{d}$, $\mu:\left[t_{0}, T\right] \times B C\left(\left[-\tau, t_{0}\right] ; \mathbb{R}^{d}\right) \rightarrow \mathbb{R}^{d}$ and $Q: B C\left(\left[-\tau, t_{0}\right] ; \mathbb{R}^{d}\right) \rightarrow \mathbb{R}^{d}$, are Borel measurable. We consider the following G-NSFDE:

$$
d\left[Y(t)-Q\left(t, Y_{t}\right)\right]=\kappa\left(t, Y_{t}\right) d t+\lambda\left(t, Y_{t}\right) d\langle B, B\rangle(t)+\mu\left(t, Y_{t}\right) d B(t)
$$

where $Y_{t}=\{Y(t+\theta):-\tau \leq \theta \leq 0, \tau>0\}$ indicates the collection of continuous bounded functions $\Lambda:\left[-\tau, t_{0}\right] \rightarrow \mathbb{R}^{d}$ equipped with norm $\|\Lambda\|=\sup _{-\tau \leq \theta \leq 0}|\Lambda(\theta)|$, which is a $B C\left(\left[-\tau, t_{0}\right] ; \mathbb{R}^{d}\right)$-valued stochastic process and $Y(t)$ is the value of stochastic process at time $t$. All the coefficients $\kappa, \lambda, \mu \in M_{G}^{2}\left([-\tau, T] ; \mathbb{R}^{k}\right)$ and $\{\langle B, B\rangle(t), t \geq 0\}$ is the quadratic variation process of G-Brownian motion $\{B(t), t \geq 0\}$. We denote by $\mathbb{L}^{2}$ the space of all $\mathcal{F}_{t}$-adapted process $Y(t), 0 \leq t \leq T$, such that $\|Y\|_{\mathbb{L}^{2}}=\sup _{-\tau \leq t \leq T}|Y(t)|<\infty$. The initial data of equation (1.1) is $\mathcal{F}_{0}$-measurable and a $B C\left(\left[-\tau, t_{0}\right] ; \mathbb{R}^{d}\right)$-valued random variable

$$
Y_{t_{0}}=\zeta=\{\zeta(\theta):-\tau<\theta \leq 0\}
$$

such that $\zeta \in M_{G}^{2}\left(\left[-\tau, t_{0}\right] ; \mathbb{R}^{d}\right)$. In the integral form the above equation is expressed as

$$
\begin{aligned}
Y(t)-Q\left(t, Y_{t}\right)= & \zeta(0)-Q\left(t_{0}, Y_{t_{0}}\right)+\int_{t_{0}}^{t} \kappa\left(s, Y_{s}\right) d s+\int_{t_{0}}^{t} \lambda\left(s, Y_{s}\right) d\langle B, B\rangle(s) \\
& +\int_{t_{0}}^{t} \mu\left(s, Y_{s}\right) d B(s) .
\end{aligned}
$$

An $\mathbb{R}^{d}$-valued stochastic processes $Y(t), t \in[-\tau, T]$, satisfying the following features, is known as the solution of equation (1.1) with initial data (1.2).

(i) The coefficients $\kappa\left(t, Y_{t}\right) \in \mathcal{L}^{1}\left([o, T] ; \mathbb{R}^{d}\right)$ and $\lambda\left(t, Y_{t}\right), \mu\left(t, Y_{t}\right) \in \mathcal{L}^{2}\left(\left[t_{0}, T\right] ; \mathbb{R}^{d}\right)$.

(ii) $Y(t)$ is $\mathcal{F}_{t}$-adapted and continuous for all $t \in\left[t_{0}, T\right]$.

(iii) $Y_{0}=\zeta$ and, for each $t \in\left[t_{0}, T\right]$,

$$
d\left[Y(t)-Q\left(Y_{t}\right)\right]=\kappa\left(t, Y_{t}\right) d t+\lambda\left(t, Y_{t}\right) d\langle B, B\rangle(t)+\mu\left(t, Y_{t}\right) d B(t) \text { q.s. }
$$


By a unique solution $Y(t)$ of G-NSFDE (1.1), we mean that it is equivalent to any other solution $Z(t)$. In other words, we have to prove that

$$
E\left[\sup _{-\tau \leq u \leq t}|Y(u)-Z(u)|^{2}\right]=0 .
$$

All through this article, the non-uniform Lipschitz condition, weakened linear growth condition and contractive condition are considered. These conditions are, respectively, given as follows.

$\left(\mathrm{H}_{\mathrm{i}}\right)$ : Let $t \in\left[t_{0}, T\right]$. For every $U, \chi \in B C\left([-\tau, 0] ; \mathbb{R}^{k}\right)$

$$
|\kappa(t, U)-\kappa(t, \chi)|^{2}+|\lambda(t, U)-\lambda(t, \chi)|^{2}+|\mu(t, U)-\mu(t, \chi)|^{2} \leq \Upsilon\left(|U-\chi|^{2}\right),
$$

where the function $\Upsilon(\cdot): \mathbb{R}^{+} \rightarrow \mathbb{R}^{+}$is non-decreasing and concave with $\Upsilon(0)=0, \Upsilon(w)>0$ for $w>0$ and

$$
\int_{0+} \frac{d w}{\Upsilon(w)}=\infty
$$

Since $\Upsilon$ is concave and $\Upsilon(0)=0$, for all $w \geq 0$,

$$
\Upsilon(w) \leq \alpha+\beta w
$$

where $\alpha$ and $\beta$ are positive constants.

$\left(\mathrm{H}_{\mathrm{ii}}\right)$ : For every $t \in\left[t_{0}, T\right]$ and $\kappa(t, 0), \lambda(t, 0), \mu(t, 0) \in L^{2}$,

$$
|\kappa(t, 0)|^{2}+|\lambda(t, 0)|^{2}+|\mu(t, 0)|^{2} \leq K
$$

where $K$ is a positive constant.

$\left(\mathrm{H}_{\mathrm{iii}}\right)$ : For every $U, \chi \in B C\left([-\tau, 0] ; \mathbb{R}^{d}\right)$ and $t \in\left[t_{0}, T\right]$,

$$
\begin{aligned}
& |Q(t, U)-Q(t, \chi)|^{2} \leq \alpha_{0}|U-\chi|^{2}, \\
& |Q(t, 0)|^{2} \leq \alpha_{0},
\end{aligned}
$$

where $0<\alpha_{0}<\frac{1}{15}$.

Just for simplicity we consider $d=1$ throughout the paper.

\section{Preliminaries}

This section is devoted to some basic literature, which will be helpful in our forthcoming research work. Firstly, we give three important inequalities. They are called Gronwall's inequality, Bihari's inequality and Hölder's inequality respectively [26].

Lemma 2.1 Let the function $\phi(t)$ be real and continuous on $[a, b]$. Let $K \geq 0$ and $\varphi(t) \geq 0$, $t \in[a, b]$. If $\phi(t) \leq K+\int_{a}^{b} \varphi(s) \phi(s) d s$, for every $a \leq t \leq b$, then

$$
\phi(t) \leq K e^{\int_{a}^{t} \varphi(s) d s},
$$

for every $a \leq t \leq b$.

The following lemma has been borrowed from [26, 27]. 
Lemma 2.2 Let the function $\Upsilon(\cdot): \mathbb{R}^{+} \rightarrow \mathbb{R}^{+}$be concave, continuous and non-decreasing satisfying $\Upsilon(0)=0$ and $\Upsilon(v)>0$ for $v>0$. Assume that $\phi(t) \geq 0$, for all $0 \leq t_{0} \leq t \leq T<\infty$, satisfies

$$
\phi(t) \leq K+\int_{t_{0}}^{t} \varphi(s) \Upsilon(\phi(s)) d s,
$$

where $K$ is a positive real number and $\varphi:\left[t_{0}, T\right] \rightarrow \mathbb{R}^{+}$. The following features hold.

(i) If $K=0$, then $\phi(t)=0, t \in\left[t_{0}, T\right]$.

(ii) If $K>0$, we define $U(t)=\int_{t_{0}}^{t} \frac{1}{\Upsilon(s)} d s$, for $t \in\left[t_{0}, T\right]$, then

$$
\phi(t) \leq U^{-1}\left(U(C)+\int_{t_{0}}^{t} \varphi(s) d s\right)
$$

where $U^{-1}$ is the inverse function of $U$.

Lemma 2.3 Iffor any $q, r>1, \frac{1}{q}+\frac{1}{r}=1$ and $\phi, \varphi \in L^{2}$ then $\phi \varphi \in L^{1}$ and

$$
\int_{a}^{b} \phi \varphi \leq\left(\int_{a}^{b}|\phi|^{q}\right)^{\frac{1}{q}}\left(\int_{a}^{b}|\varphi|^{r}\right)^{\frac{1}{r}}
$$

Next we specify some basic definitions and results of the G-expectation and G-Brownian motion [24, 28-31]. Let $\Omega$ be a nonempty basic space. Assume the space of linear realvalued functions defined on $\Omega$ is denoted by $\mathcal{H}$.

Definition 2.4 A functional $E: \mathcal{H} \rightarrow \mathbb{R}$ is named a G-expectation if the following characteristics hold:

(i) $E[Y] \leq E[Z]$ whenever $Y \leq Z$, where $Y, Z \in \mathcal{H}$.

(ii) $E[\gamma]=\gamma$, where $\gamma$ is any real constant.

(iii) $E[\theta Z]=\theta E[Z]$, for any $\theta>0$.

(iv) $E[Y+Z] \leq E[Y]+E[Z]$, where $Y, Z \in \mathcal{H}$.

In the case when $E$ holds only the characteristics (i) and (ii), then it is known as a nonlinear expectation. For each $\omega \in \Omega$ define the canonical process by $B_{t}(\omega)=\omega_{t}, t \geq 0$. The filtration generated by the canonical process $\left\{B_{t}, t \geq 0\right\}$ is defined by $\mathcal{F}_{t}=\sigma\left\{B_{s}, 0 \leq s \leq t\right\}$, $\mathcal{F}=\left\{\mathcal{F}_{t}\right\}_{t \geq 0}$. Let $\Omega$ be the space of all $R^{d}$-valued continuous paths $\left\{\omega_{t}, t \geq 0\right\}$ that start from 0 and $B$ the canonical process. Also, suppose that associated with the distance given below, $\Omega$ is a metric space,

$$
\rho\left(w^{1}, w^{2}\right)=\sum_{i=1}^{\infty} \frac{1}{2^{i}}\left(\max _{t \in[0, k]}\left|w_{t}^{1}-w_{t}^{2}\right| \wedge 1\right)
$$

Let $C_{b \text {.Lip }}\left(\mathbb{R}^{k \times d}\right)$ denote the set of bounded Lipschitz functions on $\mathbb{R}^{k \times d}$. Fix $T \geq 0$ and set

$$
L_{i p}^{0}\left(\Omega_{T}\right)=\left\{\phi\left(B_{t_{1}}, B_{t_{2}}, \ldots, B_{t_{k}}\right): k \geq 1, t_{1}, t_{2}, \ldots, t_{k} \in[0, T], \phi \in C_{b . \mathrm{Lip}}\left(\mathbb{R}^{k \times d}\right)\right\},
$$

$L_{i p}^{0}\left(\Omega_{t}\right) \subseteq L_{i p}^{0}\left(\Omega_{T}\right)$ for $t \leq T$ and $L_{i p}^{0}(\Omega)=\bigcup_{n=1}^{\infty} L_{i p}^{0}\left(\Omega_{n}\right)$. The completion of $L_{i p}^{0}(\Omega)$ under the Banach norm $E\left[|\cdot|^{p}\right]^{\frac{1}{p}}, p \geq 1$, is denoted by $L_{G}^{p}(\Omega)$, where $L_{G}^{p}\left(\Omega_{t}\right) \subseteq L_{G}^{p}\left(\Omega_{T}\right) \subseteq L_{G}^{p}(\Omega)$ 
for $0 \leq t \leq T<\infty$. Suppose $\pi_{T}=\left\{t_{0}, t_{1}, \ldots, t_{N}\right\}, 0 \leq t_{0} \leq t_{1} \leq \cdots \leq t_{N} \leq \infty$ is a partition of $[0, T]$. Set $p \geq 1$, then $M_{G}^{p, 0}(0, T)$ indicates a collection of the following type of processes:

$$
\eta_{t}(w)=\sum_{i=0}^{N-1} \xi_{i}(w) I_{\left[t_{i}, t_{i+1}\right]}(t),
$$

where $\xi_{i} \in L_{G}^{p}\left(\Omega_{t_{i}}\right), i=0,1, \ldots, N-1$. Furthermore, the completion of $M_{G}^{p, 0}(0, T)$ with the norm given below is indicated by $M_{G}^{p}(0, T), p \geq 1$,

$$
\|\eta\|=\left\{\int_{0}^{T} E\left[\left|\eta_{s}\right|^{p}\right] d s\right\}^{1 / p}
$$

Definition 2.5 The canonical process $\{B(t)\}_{t \geq 0}$ under the G-expectation $E$ defined on $L_{i p}^{0}(\Omega)$ and satisfying the following properties is called a G-Brownian motion:

(1) $B(0)=0$.

(2) The increment $B_{t+k}-B_{t}$, for any $t, k \geq 0$, is G-normally distributed and independent from $B_{t_{1}}, B_{t_{2}}, \ldots, B_{t_{n}}$, for $n \in N$ and $0 \leq t_{1} \leq t_{2} \leq \cdots \leq t_{n} \leq t$.

In the G-framework the Itô integral $I(\eta)$ and the corresponding quadratic variation process $\left\{\langle B\rangle_{t}\right\}_{t \geq 0}$ are, respectively, defined by

$$
\begin{aligned}
& I(\eta)=\int_{0}^{T} \eta_{u} d B_{u}=\sum_{i=0}^{N-1} \delta_{i}\left(B_{t_{i+1}}-B_{t_{i}}\right), \\
& \langle B\rangle_{t}=B_{t}^{2}-2 \int_{0}^{t} B_{u} d B_{u} .
\end{aligned}
$$

The capacity $\hat{c}(\cdot)$ associated to a weakly compact collection of probability measures $\mathcal{P}$ is given by

$$
\hat{c}(D)=\sup _{P \in \mathcal{P}} P(D), \quad D \in \mathcal{B}(\Omega)
$$

where $\mathcal{B}(\Omega)$ is the Borel $\sigma$-algebra of $\Omega . D$ is known as polar set if it has zero capacity, that is, $\hat{c}(D)=0$ and a characteristic holds quasi-surely in short (q.s.) if it is valid outside a polar set. For each $Y \in L^{0}\left(\Omega_{T}\right)$, the G-expectation $E$ is given by $E[Y]=\sup _{P \in \mathcal{P}} E_{P}[Y]$, where for each $P \in \mathcal{P}, E_{P}[Y]$ exists. For more details of the above definition, we refer the reader to [28].

\section{Some important lemmas}

Firstly, we define the Picard iterations sequence as follows. Let, for $t \in\left[t_{0}, T\right], Y^{0}(t)=\zeta(0)$ and $Y_{t_{0}}^{k}=\zeta$ for each $k=1,2, \ldots$, then

$$
\begin{aligned}
Y^{k}(t)= & Q\left(t, Y_{t}^{k}\right)-Q\left(t, Y_{t_{0}}^{k}\right)+\zeta(0)+\int_{t_{0}}^{t} \kappa\left(s, Y_{s}^{k-1}\right) d s+\int_{t_{0}}^{t} \lambda\left(s, Y_{s}^{k-1}\right) d\langle B, B\rangle(s) \\
& +\int_{t_{0}}^{t} \mu\left(s, Y_{s}^{k-1}\right) d B(s), \quad t \in\left[t_{0}, T\right] .
\end{aligned}
$$


In the following lemma we show that $Y^{k}(t) \in M_{G}^{2}\left([-\tau, T] ; \mathbb{R}^{k}\right)$. Then we prove another important lemma. These lemmas will be used in the existence-and-uniqueness theorem.

Lemma 3.1 Suppose that assumptions $\mathrm{H}_{\mathrm{i}}, \mathrm{H}_{\mathrm{ii}}$ and $\mathrm{H}_{\mathrm{iii}}$ are satisfied. Then, for all $k \geq 1$,

$$
\sup _{-\tau \leq t \leq T} E\left|Y^{k}(t)\right|^{2} \leq \alpha
$$

where $\alpha=\frac{K_{2}}{1-15 \alpha_{0}} e^{\frac{10 b \alpha^{*}}{1-15 \alpha_{0}}\left(T-t_{0}\right)}, K_{2}=\left[1+15 \alpha_{0}+10 b \alpha^{*}\left(T-t_{0}\right)\right] E|\zeta|^{2}+K_{1}, K_{1}=5\left[1+3 \alpha_{0}\right] E|\zeta|^{2}+$ $60 \alpha_{0}+10 K\left(T-t_{0}\right) \alpha^{*}(K+a), \alpha^{*}=\alpha_{1}+\alpha_{2}+\alpha_{3}, \alpha_{1}, \alpha_{2}$ and $\alpha_{3}$ are positive constants.

Proof It is obvious that $Y^{0}(\cdot) \in M_{G}^{2}\left([-\tau, T] ; \mathbb{R}^{k}\right)$. Using the basic inequality $\mid c_{1}+c_{2}+c_{3}+$ $c_{4}+\left.c_{5}\right|^{2} \leq 5\left|c_{1}\right|^{2}+5\left|c_{2}\right|^{2}+5\left|c_{3}\right|^{2}+5\left|c_{4}\right|^{2}+5\left|c_{5}\right|^{2}$, equation (3.1) follows;

$$
\begin{aligned}
\left|Y^{k}(t)\right|^{2} \leq & 5|\zeta(0)|^{2}+5\left|Q\left(t, Y_{t}^{k}\right)-Q\left(t_{0}, Y_{t_{0}}^{k}\right)\right|^{2}+5\left|\int_{t_{0}}^{t} \kappa\left(s, Y_{s}^{k-1}\right) d s\right|^{2} \\
& +5\left|\int_{t_{0}}^{t} \lambda\left(s, Y_{s}^{k-1}\right) d\langle B, B\rangle(s)\right|^{2}+5\left|\int_{t_{0}}^{t} \mu\left(s, Y_{s}^{k-1}\right) d B(s)\right|^{2}
\end{aligned}
$$

By taking G-expectation on both sides, using Lemma 2.3 and the BDG inequalities [16] we obtain

$$
\begin{aligned}
& E\left|Y^{k}(t)\right|^{2} \leq 5 E|\zeta(0)|^{2}+5 E\left|Q\left(t, Y_{t}^{k}\right)-Q\left(t_{0}, Y_{t_{0}}^{k}\right)\right|^{2}+5 \alpha_{1} E \int_{t_{0}}^{t}\left|\kappa\left(s, Y_{s}^{k-1}\right)\right|^{2} d s \\
& +5 \alpha_{2} E \int_{t_{0}}^{t}\left|\lambda\left(s, Y_{s}^{k-1}\right)\right|^{2} d s+5 \alpha_{3} \int_{t_{0}}^{t}\left|\mu\left(s, Y_{s}^{k-1}\right)\right|^{2} d s \\
& \leq 5 E|\zeta(0)|^{2} \\
& +5 E\left|Q\left(t, Y_{t}^{k}\right)-Q(t, 0)+Q\left(t_{0}, 0\right)-Q\left(t_{0}, Y_{t_{0}}^{k}\right)+Q(t, 0)-Q\left(t_{0}, 0\right)\right|^{2} \\
& +10 \alpha_{1} E \int_{t_{0}}^{t}\left(\left|\kappa\left(s, Y_{s}^{k-1}\right)-\kappa(s, 0)\right|^{2}+|\kappa(s, 0)|^{2}\right) d s \\
& +10 \alpha_{2} E \int_{t_{0}}^{t}\left(\left|\lambda\left(s, Y_{s}^{k-1}\right)-\lambda(s, 0)\right|^{2}+|\lambda(s, 0)|^{2}\right) d(s) \\
& +10 \alpha_{3} \int_{t_{0}}^{t}\left(\left|\mu\left(s, Y_{s}^{k-1}\right)-\mu(s, 0)\right|^{2}+|\mu(s, 0)|^{2}\right) d(s) \\
& \leq 5 E|\zeta(0)|^{2}+15 E\left|Q\left(t, Y_{t}^{k}\right)-Q(t, 0)\right|^{2}+15 E\left|Q\left(t_{0}, 0\right)-Q\left(t_{0}, Y_{t_{0}}^{k}\right)\right|^{2} \\
& +30 E|Q(t, 0)|^{2}+30 E\left|Q\left(t_{0}, 0\right)\right|^{2}+10 \alpha_{1} E \int_{t_{0}}^{t}|\kappa(s, 0)|^{2} d s \\
& +10 \alpha_{1} E \int_{t_{0}}^{t}\left|\kappa\left(s, Y_{s}^{k-1}\right)-\kappa(s, 0)\right|^{2} d s \\
& +10 \alpha_{2} E \int_{t_{0}}^{t}|\lambda(s, 0)|^{2} d s+10 \alpha_{2} E \int_{t_{0}}^{t}\left|\lambda\left(s, Y_{s}^{k-1}\right)-\lambda(s, 0)\right|^{2} d(s) \\
& +10 \alpha_{3} \int_{t_{0}}^{t}|\mu(s, 0)|^{2} d(s)+10 \alpha_{3} \int_{t_{0}}^{t}\left|\mu\left(s, Y_{s}^{k-1}\right)-\mu(s, 0)\right|^{2} d(s) \text {. }
\end{aligned}
$$


By using assumptions $\mathrm{H}_{\mathrm{i}}, \mathrm{H}_{\mathrm{ii}}$ and $\mathrm{H}_{\mathrm{iii}}$, we have

$$
\begin{aligned}
E\left|Y^{k}(t)\right|^{2} \leq & 5 E|\zeta(0)|^{2}+15 \alpha_{0} E\left|Y_{t}^{k}\right|^{2}+15 \alpha_{0} E\left|Y_{t_{0}}^{k}\right|^{2}+60 \alpha_{0} \\
& +10 \alpha_{1} K\left(T-t_{0}\right)+10 \alpha_{2} K\left(T-t_{0}\right)+10 \alpha_{3} K\left(T-t_{0}\right) \\
& +10 \alpha_{1} E \int_{t_{0}}^{t} \Upsilon\left(\left|Y_{s}^{k-1}\right|^{2}\right) d s+10 \alpha_{2} E \int_{t_{0}}^{t} \Upsilon\left(\left|Y_{s}^{k-1}\right|^{2}\right) d s \\
& +10 \alpha_{3} \int_{t_{0}}^{t} \Upsilon\left(\left|Y_{s}^{k-1}\right|^{2}\right) d(s) \\
= & 5 E|\zeta(0)|^{2}+60 \alpha_{0}+10 K\left(T-t_{0}\right)\left(\alpha_{1}+\alpha_{2}+\alpha_{3}\right) \\
& +15 \alpha_{0} E\left|Y_{t}^{k}\right|^{2}+15 \alpha_{0} E\left|Y_{t_{0}}^{k}\right|^{2}+10\left(\alpha_{1}+\alpha_{2}+\alpha_{3}\right) E \int_{t_{0}}^{t} \Upsilon\left(\left|Y_{s}^{k-1}\right|^{2}\right) d s \\
\leq & 5 E|\zeta(0)|^{2}+60 \alpha_{0}+10 K\left(T-t_{0}\right)\left(\alpha_{1}+\alpha_{2}+\alpha_{3}\right)+10 \alpha\left(\alpha_{1}+\alpha_{2}+\alpha_{3}\right)\left(T-t_{0}\right) \\
& +15 \alpha_{0} E\left|Y_{t}^{k}\right|^{2}+15 \alpha_{0} E|\zeta|^{2}+10 \beta\left(\alpha_{1}+\alpha_{2}+\alpha_{3}\right) E \int_{t_{0}}^{t}\left|Y_{s}^{k-1}\right|^{2} d s \\
= & K_{1}+15 \alpha_{0} E\left|Y_{t}^{k}\right|^{2}+10 b\left(\alpha_{1}+\alpha_{2}+\alpha_{3}\right) E \int_{t_{0}}^{t}\left|Y_{s}^{k-1}\right|^{2} d s
\end{aligned}
$$

where $K_{1}=5 E|\zeta(0)|^{2}+60 \alpha_{0}+10 K\left(T-t_{0}\right)\left(\alpha_{1}+\alpha_{2}+\alpha_{3}\right)+10 \alpha\left(\alpha_{1}+\alpha_{2}+\alpha_{3}\right)\left(T-t_{0}\right)+$ $15 \alpha_{0} E|\zeta|^{2}$. We note that

$$
\sup _{t_{0} \leq s \leq t}\left|Y_{s}^{k}\right|^{2} \leq \sup _{-\tau \leq q \leq t}\left|Y^{k}(q)\right|^{2} \leq|\zeta|^{2}+\sup _{t_{0} \leq q \leq t}\left|Y^{k}(q)\right|^{2}
$$

from which follows

$$
\begin{aligned}
\sup _{-\tau \leq q \leq t} E\left|Y^{k}(q)\right|^{2} \leq & E|\zeta|^{2}+K_{1}+15 \alpha_{0} \sup _{-\tau \leq q \leq t} E\left|Y^{k}(q)\right|^{2} \\
& +10 \beta\left(\alpha_{1}+\alpha_{2}+\alpha_{3}\right) E \int_{t_{0}}^{t} \sup _{-\tau \leq q \leq t}\left|Y^{k-1}(q)\right|^{2} d s .
\end{aligned}
$$

For any $j \geq 1$, we observe that

$$
\max _{1 \leq k \leq j} E\left|Y_{s}^{k-1}\right|^{2} \leq E|\zeta|^{2}+\max _{1 \leq k \leq j} E\left|Y^{k}(s)\right|^{2}
$$

and we obtain

$$
\begin{aligned}
\max _{1 \leq k \leq j} \sup _{-\tau \leq q \leq t} E\left|Y^{k}(q)\right|^{2} \leq & E|\zeta|^{2}+K_{1}+15 \alpha_{0} \sup _{-\tau \leq q \leq t} E\left|Y^{k}(q)\right|^{2} \\
& +10 \beta\left(\alpha_{1}+\alpha_{2}+\alpha_{3}\right) E \int_{t_{0}}^{t}\left[E|\zeta|^{2}+\max _{1 \leq k \leq j} \sup _{-\tau \leq q \leq t} E\left|Y^{k}(q)\right|^{2}\right] d s \\
\leq & K_{2}+15 \alpha_{0} \sup _{-\tau \leq q \leq t} E\left|Y^{k}(q)\right|^{2} \\
& +10 \beta\left(\alpha_{1}+\alpha_{2}+\alpha_{3}\right) E \int_{t_{0}}^{t} \max _{1 \leq k \leq j} \sup _{-\tau \leq q \leq t} E\left|Y^{k}(q)\right|^{2} d s,
\end{aligned}
$$


where $K_{2}=E|\zeta|^{2}+K_{1}+10 \beta\left(\alpha_{1}+\alpha_{2}+\alpha_{3}\right)\left(T-t_{0}\right) E|\zeta|^{2}$. We have

$$
\max _{1 \leq k \leq j} \sup _{-\tau \leq q \leq t} E\left|Y^{k}(q)\right|^{2} \leq \frac{K_{2}}{1-15 \alpha_{0}}+\frac{10 \beta\left(\alpha_{1}+\alpha_{2}+\alpha_{3}\right)}{1-15 \alpha_{0}} \int_{t_{0}}^{t} \max _{1 \leq k \leq j} \sup _{-\tau \leq q \leq t} E\left|Y^{k}(q)\right|^{2} d s .
$$

Consequently, the Gronwall inequality gives

$$
\max _{1 \leq k \leq j-\tau \leq q \leq t} \sup _{-} E\left|Y^{k}(t)\right|^{2} \leq \alpha
$$

where $\alpha=\frac{K_{2}}{1-15 \alpha_{0}} e^{\frac{10 \beta\left(\alpha_{1}+\alpha_{2}+\alpha_{3}\right)}{1-15 \alpha_{0}}\left(T-t_{0}\right)}$, but $j$ is arbitrary, so

$$
\sup _{-\tau \leq t \leq T} E\left|Y^{k}(t)\right|^{2} \leq \alpha
$$

The proof is complete.

Lemma 3.2 Let hypotheses $\mathrm{H}_{\mathrm{i}}, \mathrm{H}_{\mathrm{ii}}$ and $\mathrm{H}_{\mathrm{iii}}$ hold. Then for every $k, d \geq 1$, a constant $\beta>0$ exists such that

$$
\begin{aligned}
E\left[\sup _{-\tau \leq s \leq t}\left|Y^{k+d}(s)-Y^{k}(s)\right|^{2}\right] & \leq \beta \int_{t_{0}}^{t} \Upsilon\left(E\left[\sup _{-\tau \leq q \leq s}\left|Y^{k+d-1}(q)-Y^{k-1}(q)\right|^{2}\right]\right) d s \\
& \leq \gamma\left(t-t_{0}\right),
\end{aligned}
$$

where $\gamma=\beta \Upsilon(4 C)$ and $\beta=\frac{4\left(\alpha_{1}+\alpha_{2}+\alpha_{3}\right)}{1-4 \alpha_{0}}$.

Proof By the basic inequality $\left|c_{1}+c_{2}+c_{3}+c_{4}\right|^{2} \leq 4\left|c_{1}\right|^{2}+4\left|c_{2}\right|^{2}+4\left|c_{3}\right|^{2}+4\left|c_{4}\right|^{2}$, equation (3.1) yields

$$
\begin{aligned}
\left|Y^{k+d}(t)-Y^{k}(t)\right|^{2} \leq & 4\left|Q\left(t, Y_{t}^{k+d}\right)-Q\left(t, Y_{t}^{k}\right)\right|^{2}+4\left|\int_{t_{0}}^{t}\left[\kappa\left(s, Y_{s}^{k+d-1}\right)-\kappa\left(s, Y_{s}^{k-1}\right)\right] d s\right|^{2} \\
& +4\left|\int_{t_{0}}^{t}\left[\lambda\left(s, Y_{s}^{k+d-1}\right)-\lambda\left(s, Y_{s}^{k-1}\right)\right] d\langle B, B\rangle(s)\right|^{2} \\
& +4\left|\int_{t_{0}}^{t}\left[\mu\left(s, Y_{s}^{k+d-1}\right)-\mu\left(s, Y_{s}^{k-1}\right)\right] d B(s)\right|^{2}
\end{aligned}
$$

Next, on both sides, we take sub-expectations. Then we apply the Jensen inequality $E(w(z)) \leq w(E(z))$, BDG inequalities [16] and hypotheses $\mathrm{H}_{\mathrm{i}}, \mathrm{H}_{\mathrm{ii}}, \mathrm{H}_{\mathrm{iii}}$ to find

$$
\begin{aligned}
& E\left[\sup _{-\tau \leq s \leq t}\left|Y^{k+d}(s)-Y^{k}(s)\right|^{2}\right] \\
& \leq 4 \alpha_{0} E\left[\sup _{-\tau \leq q \leq t}\left|Y^{k+d}(q)-Y^{k}(q)\right|^{2}\right] \\
&+4 \alpha_{1} \int_{t_{0}}^{t} \Upsilon\left(E\left[\sup _{-\tau \leq q \leq s}\left|Y^{k+d-1}(q)-Y^{k-1}(q)\right|^{2}\right]\right) d s \\
&+4 \alpha_{2} \int_{t_{0}}^{t} \Upsilon\left(E\left[\sup _{-\tau \leq q \leq s}\left|Y^{k+d-1}(q)-Y^{k-1}(q)\right|^{2}\right]\right) d s \\
&+4 \alpha_{3} \int_{t_{0}}^{t} \Upsilon\left(E\left[\sup _{-\tau \leq q \leq s}\left|Y^{k+d-1}(q)-Y^{k-1}(q)\right|^{2}\right]\right) d s
\end{aligned}
$$




$$
\begin{aligned}
& \leq 4 \alpha_{0} E\left[\sup _{-\tau \leq q \leq t}\left|Y^{k+d}(q)-Y^{k}(q)\right|^{2}\right] \\
&+4\left(\alpha_{1}+\alpha_{2}+\alpha_{3}\right) \int_{t_{0}}^{t} \Upsilon\left(E\left[\sup _{-\tau \leq q \leq s}\left|Y^{k+d-1}(q)-Y^{k-1}(q)\right|^{2}\right]\right) d s, \\
& E\left[\sup _{-\tau \leq s \leq t}\left|Y^{k+d}(s)-Y^{k}(s)\right|^{2}\right] \leq \beta \int_{t_{0}}^{t} \Upsilon\left(E\left[\sup _{-\tau \leq q \leq s}\left|Y^{k+d-1}(q)-Y^{k-1}(q)\right|^{2}\right]\right) d s,
\end{aligned}
$$

where $\beta=\frac{4\left(\alpha_{1}+\alpha_{2}+\alpha_{3}\right)}{1-4 \alpha_{0}}$. Finally, we use Lemma 3.1 and get

$$
E\left[\sup _{-\tau \leq s \leq t}\left|Y^{k+d}(s)-Y^{k}(s)\right|^{2}\right] \leq \beta \Upsilon(4 C)\left(t-t_{0}\right)=\gamma\left(t-t_{0}\right),
$$

where $\gamma=\beta \Upsilon(4 C)$. The proof is complete.

\section{Existence-and-uniqueness results for G-NSFDEs}

In this section first we construct a key lemma. We set

$$
\phi_{1}(t)=\gamma\left(t-t_{0}\right), \quad t \in\left[t_{0}, T\right]
$$

Let us define a recursive function as follows. For every $l, d \geq 1$,

$$
\begin{aligned}
& \phi_{k+1}(t)=\beta \int_{t_{0}}^{t} \Upsilon\left(\phi_{k}(s)\right) d s, \\
& \phi_{k, d}(t)=E\left[\sup _{-\tau \leq q \leq s}\left|Y^{k+d}(q)-Y^{k}(q)\right|^{2}\right] .
\end{aligned}
$$

Select $T_{1} \in\left[t_{0}, T\right]$ such that

$$
\beta \Upsilon\left(\gamma\left(t-t_{0}\right)\right) \leq \gamma
$$

for all $t \in\left[t_{0}, T_{1}\right]$.

Lemma 4.1 Let hypotheses $\mathrm{H}_{\mathrm{i}}, \mathrm{H}_{\mathrm{ii}}$ and $\mathrm{H}_{\mathrm{iii}}$ hold. Then, for all $d \geq 1$ and any $k \geq 1$, a positive $T_{1} \in\left[t_{0}, T\right]$ exists such that

$$
0 \leq \phi_{k, d}(t) \leq \phi_{k}(t) \leq \phi_{k-1}(t) \leq \cdots \leq \phi_{1}(t)
$$

for all $t \in\left[t_{0}, T\right]$.

Proof Mathematical induction is used to prove the inequality (4.4). We use Lemma 3.2 and the definition of the function $\phi(\cdot)$ to obtain

$$
\begin{aligned}
\phi_{1, d}(t) & =E\left[\sup _{-\tau \leq q \leq s}\left|Y^{1+d}(q)-Y^{1}(q)\right|^{2}\right] \leq \gamma\left(t-t_{0}\right)=\phi_{1}(t), \\
\phi_{2, d}(t) & =E\left[\sup _{-\tau \leq q \leq s}\left|Y^{2+d}(q)-Y^{2}(q)\right|^{2}\right] \leq \beta \int_{t_{0}}^{t} \Upsilon\left(E\left[\sup _{-\tau \leq q \leq s}\left|Y^{1+d}(q)-Y^{1}(q)\right|^{2}\right]\right) d s \\
& \leq \beta \int_{t_{0}}^{t} \Upsilon\left(\phi_{1}(s)\right) d s=\phi_{2}(t) .
\end{aligned}
$$


By (4.3), we obtain

$$
\phi_{2}(t)=\beta \int_{t_{0}}^{t} \Upsilon\left(\phi_{1}(s)\right) d s=\int_{t_{0}}^{t} \beta \Upsilon\left(\gamma\left(t-t_{0}\right)\right) d s \leq \gamma\left(t-t_{0}\right)=\phi_{1}(t) .
$$

Hence for every $t \in\left[t_{0}, T_{1}\right]$, we derive that $\phi_{2, d}(t) \leq \phi_{2}(t) \leq \phi_{1}(t)$. Assume that (4.4) is satisfied for some $k \geq 1$. Then we need to verify that the inequality (4.4) holds for $k+1$. We proceed as follows:

$$
\begin{aligned}
\phi_{k+1, d}(t) & =E\left[\sup _{-\tau \leq q \leq s}\left|Y^{k+d+1}(q)-Y^{k+1}(q)\right|^{2}\right] \\
& \leq \beta \int_{t_{0}}^{t} \Upsilon\left(E\left[\sup _{-\tau \leq q \leq s}\left|Y^{k+d}(q)-Y^{k}(q)\right|^{2}\right]\right) d s \\
& =\beta \int_{t_{0}}^{t} \Upsilon\left(\phi_{k, d}(s)\right) d s \\
& \leq \beta \int_{t_{0}}^{t} \Upsilon\left(\phi_{k}(s)\right) d s \\
& =\phi_{k+1}(t) .
\end{aligned}
$$

Also

$$
\phi_{k+1}(t)=\beta \int_{t_{0}}^{t} \Upsilon\left(\phi_{k}(s)\right) d s \leq \beta \int_{t_{0}}^{t} \Upsilon\left(\phi_{k-1}(s)\right) d s=\phi_{k}(s) .
$$

Hence for all $t \in\left[t_{0}, T_{1}\right], \phi_{k+1, d}(t) \leq \phi_{k+1}(t) \leq \phi_{k}(s)$, that is, Lemma 4.1 is true for $k+1$. The proof is completed.

Theorem 4.2 Suppose hypotheses $\mathrm{H}_{\mathrm{i}}, \mathrm{H}_{\mathrm{ii}}$ and $\mathrm{H}_{\mathrm{iii}}$ are valid. Then there exists at most one solution of the G-NSFDE (1.1) having initial data (1.2).

Proof Firstly, we derive the uniqueness of solution. Let the G-NSFDE (1.1) with initial condition (1.2) admit two solutions $Y(t)$ and $Z(t)$. Then we get

$$
\begin{aligned}
|Y(t)-Z(t)| \leq & \left|Q\left(t, Y_{t}\right)-Q\left(t, Z_{t}\right)\right|+\int_{t_{0}}^{t}\left|\kappa\left(s, Y_{s}\right)-\kappa\left(s, Z_{s}\right)\right| d s \\
& +\int_{t_{0}}^{t}\left|\lambda\left(s, Y_{s}\right)-\lambda\left(s, Z_{s}\right)\right| d\langle B, B\rangle(s)+\int_{t_{0}}^{t}\left|\mu\left(s, Y_{s}\right)-\mu\left(s, Z_{s}\right)\right| d B(s) .
\end{aligned}
$$

On both sides, we take G-expectations and use the basic inequality $\left(c_{1}+c_{2}+c_{3}+c_{4}\right)^{2} \leq$ $4\left(c_{1}^{2}+c_{2}^{2}+c_{3}^{2}+c_{4}^{2}\right)$. Then applying the Hölder inequality and the BDG inequalities we get

$$
\begin{aligned}
E|Y(t)-Z(t)|^{2} \leq & 4 E\left|Q\left(t, Y_{t}\right)-Q\left(t, Z_{t}\right)\right|^{2}+4 \alpha_{1} \int_{t_{0}}^{t} E\left|\kappa\left(s, Y_{s}\right)-\kappa\left(s, Z_{s}\right)\right|^{2} d s \\
& +4 \alpha_{2} \int_{t_{0}}^{t} E\left|\lambda\left(s, Y_{s}\right)-\lambda\left(s, Z_{s}\right)\right|^{2} d s+4 \alpha_{3} \int_{t_{0}}^{t} E\left|\mu\left(s, Y_{s}\right)-\mu\left(s, Z_{s}\right)\right| d s
\end{aligned}
$$


Using assumptions $\mathrm{H}_{\mathrm{i}}, \mathrm{H}_{\mathrm{ii}}$ and $\mathrm{H}_{\mathrm{iii}}$ we have

$$
\begin{aligned}
E\left[\sup _{-\tau<q \leq t}|Y(q)-Z(q)|^{2}\right] \leq & 4 \alpha_{0} E\left[\sup _{-\tau<q \leq t}|Y(q)-Z(q)|^{2}\right] \\
& +4\left(\alpha_{1}+\alpha_{2}+\alpha_{3}\right) \int_{t_{0}}^{t} \Upsilon\left(E\left[\sup _{-\tau<q \leq t}|Y(q)-Z(q)|^{2}\right]\right) d s,
\end{aligned}
$$

and it follows that

$$
E\left[\sup _{-\tau<q \leq t}|Y(q)-Z(q)|^{2}\right] \leq \frac{4\left(\alpha_{1}+\alpha_{2}+\alpha_{3}\right)}{1-4 \alpha_{0}} \int_{t_{0}}^{t} \Upsilon\left(E\left[\sup _{-\tau<q \leq t}|Y(q)-Z(q)|^{2}\right]\right) d s
$$

Consequently, Lemma 2.2 yields $E\left[\sup _{-\tau<q \leq t}|Y(q)-Z(q)|^{2}\right]=0, t \in\left[t_{0}, T\right]$. The uniqueness proof is completed. To show existence we note that $\phi_{k}(t)$ is continuous on $t \in\left[t_{0}, T_{1}\right]$ and decreasing on $t \in\left[t_{0}, T_{1}\right]$ for $k \geq 1$. We now use the dominated convergence theorem to define the function $\phi(t)$ as follows:

$$
\phi(t)=\lim _{k \rightarrow \infty} \phi_{k}(t)=\lim _{k \rightarrow \infty} \beta \int_{t_{0}}^{t} \Upsilon\left(\phi_{k-1}(s)\right) d s=\beta \int_{t_{0}}^{t} \Upsilon(\phi(s)) d s, \quad t_{0} \leq t \leq T_{1} .
$$

So,

$$
\phi(t) \leq \phi(0)+\beta \int_{t_{0}}^{t} \Upsilon(\phi(s)) d s
$$

Hence for every $t_{0} \leq t \leq T_{1}$, Lemma 2.2 yields $\phi(t)=0$. For all $t \in\left[t_{0}, T_{1}\right]$, from Lemma 4.1 it follows that $\phi_{k, d}(s) \leq \phi_{k}(s) \rightarrow 0$ as $k \rightarrow \infty$, which gives $E\left|Y^{k+d}(t)-Y^{k}(t)\right|^{2} \rightarrow 0$ as $k \rightarrow$ $\infty$. Then from the completeness of $L^{2}$ and assumptions $\mathrm{H}_{\mathrm{i}}, \mathrm{H}_{\mathrm{ii}}, \mathrm{H}_{\mathrm{iii}}$ follows that, for all $t \in\left[t_{0}, T_{1}\right]$,

$$
\begin{aligned}
& Q\left(t, Y_{t}^{k}\right) \rightarrow Q\left(t, Y_{t}\right), \quad \kappa\left(t, Y_{t}^{k}\right) \rightarrow \kappa\left(t, Y_{t}\right), \quad \lambda\left(t, Y_{t}^{k}\right) \rightarrow \lambda\left(t, Y_{t}\right), \\
& \mu\left(t, Y_{t}^{k}\right) \rightarrow \mu\left(t, Y_{t}\right) \quad \text { in } L^{2} \text { as } k \rightarrow \infty
\end{aligned}
$$

Hence, for all $t \in\left[t_{0}, T_{1}\right]$,

$$
\begin{aligned}
\lim _{k \rightarrow \infty} Y^{k}(t)= & \lim _{l \rightarrow \infty} Q\left(t, Y_{t}^{k}\right)-Q(t, \zeta)+\zeta(0)+\lim _{k \rightarrow \infty} \int_{t_{0}}^{t} \kappa\left(s, Y_{s}^{k-1}\right) d s \\
& +\lim _{k \rightarrow \infty} \int_{t_{0}}^{t} \lambda\left(s, Y_{s}^{k-1}\right) d\langle B, B\rangle(s)+\lim _{k \rightarrow \infty} \int_{t_{0}}^{t} \mu\left(s, Y_{s}^{k-1}\right) d B(s)
\end{aligned}
$$

that is,

$$
\begin{aligned}
Y(t)= & Q\left(t, Y_{t}\right)-Q(t, \zeta)+\zeta(0)+\int_{t_{0}}^{t} \kappa\left(s, Y_{s}\right) d s \\
& +\int_{t_{0}}^{t} \lambda\left(s, Y_{s}\right) d\langle B, B\rangle(s)+\int_{t_{0}}^{t} \mu\left(s, Y_{s}\right) d B(s)
\end{aligned}
$$

Hence the G-NSFDE (1.1) having initial data (1.2) admits a unique solution $Y(t)$ on $t \in$ $\left[t_{0}, T_{1}\right]$. By iteration, we see that equation (1.1) admits at most one solution on $t \in\left[t_{0}, T\right]$. The proof is completed. 


\section{Mean square stability}

In this section, we study the mean square stability for stochastic dynamical system (1.1). The following definition is borrowed from $[26,27]$.

Definition 5.1 Let $Y(t)$ and $Z(t)$ be any two solutions of the G-NSFDE (1.1) having the respective initial conditions $\zeta$ and $\xi$ belong to $M^{2}\left([-\tau, 0]: \mathbb{R}^{l}\right)$. A solution $Y(t)$ of equation (1.1) having initial data (1.2) is known to be mean square stable if for every $\varepsilon>0$ a $\delta(\varepsilon)>0$ exists so that $E|\zeta-\xi|^{2} \leq \delta(\varepsilon)$ implies $E|Y(t)-Z(t)|^{2}<\varepsilon$ for every $t \geq 0$.

Theorem 5.2 Suppose hypotheses $\mathrm{H}_{\mathrm{i}}$ and $\mathrm{H}_{\mathrm{ii}}$ are satisfied. Let equation (1.1) admit two solutions $Y(t)$ and $Z(t)$ with initial data $\zeta$ and $\xi$, respectively. Let $t \in[0, T]$. Iffor all $\varepsilon>0$ a $\delta(\varepsilon)>0$ exists such that $E|\zeta-\xi|^{2}<\delta(\varepsilon)$, then

$$
E|Z(t)-Y(t)|^{2} \leq \varepsilon
$$

Proof Let system (1.1) admit two solutions $Y(t)$ and $Z(t)$. Then, for any $t \in[0, T]$, it follows that

$$
\begin{aligned}
Y(t)= & \zeta(0)-Q\left(t_{0}, \zeta\right)+Q\left(t, Y_{t}\right)+\int_{t_{0}}^{t} \kappa\left(s, Y_{s}\right) d s+\int_{t_{0}}^{t} \lambda\left(s, Y_{s}\right) d\langle B, B\rangle(s) \\
& +\int_{t_{0}}^{t} \mu\left(s, Y_{s}\right) d B(s), \\
Z(t)= & \xi(0)-Q\left(t_{0}, \xi\right)+Q\left(t, Z_{t}\right)+\int_{t_{0}}^{t} \kappa\left(s, Z_{s}\right) d s+\int_{t_{0}}^{t} \lambda\left(s, Z_{s}\right) d\langle B, B\rangle(s) \\
& +\int_{t_{0}}^{t} \mu\left(s, Z_{s}\right) d B(s) .
\end{aligned}
$$

Then

$$
\begin{aligned}
Y(t)-Z(t)= & \zeta(0)-\xi(0)-Q\left(t_{0}, \zeta\right)+Q\left(t_{0}, \xi\right)+Q\left(t, Y_{t}\right)-Q\left(t, Z_{t}\right) \\
& +\int_{t_{0}}^{t}\left[\kappa\left(s, Y_{s}\right)-\kappa\left(s, Z_{s}\right)\right] d s+\int_{t_{0}}^{t}\left[\lambda\left(s, Y_{s}\right)-\lambda\left(s, Z_{s}\right)\right] d\langle B, B\rangle(s) \\
& +\int_{t_{0}}^{t}\left[\mu\left(s, Y_{s}\right)-\mu\left(s, Z_{s}\right)\right] d B(s) \quad \text { q.s. }
\end{aligned}
$$

We use the fundamental inequality $\left(c_{1}+c_{2}+c_{3}+c_{4}+c_{5}+c_{6}\right)^{2} \leq 6\left(c_{1}^{2}+c_{2}^{2}+c_{3}^{2}+c_{4}^{2}+c_{5}^{2}+\right.$ $\left.c_{6}^{2}\right)$ and the sub-expectation on both sides. Then using the Hölder inequality and BDG inequalities [16] to obtain

$$
\begin{aligned}
E\left[\sup _{-\tau \leq r \leq t}|Z(r)-Y(r)|^{2}\right] \leq & 6 E|\zeta-\xi|^{2} \\
& +6 \alpha_{0} E\left[\sup _{-\tau \leq r \leq t}|Z(r)-Y(r)|^{2}\right]+6 \alpha_{0} E|\zeta-\xi|^{2} \\
& +6\left(\alpha_{1}+\alpha_{2}+\alpha_{3}\right) \int_{t_{0}}^{t} \Upsilon\left(E\left[\sup _{-\tau \leq r \leq s}|Z(r)-Y(r)|^{2}\right]\right) d s
\end{aligned}
$$


It follows that

$$
\begin{aligned}
E\left[\sup _{-\tau \leq r \leq t}|Z(r)-Y(r)|^{2}\right] \leq & \frac{6\left(1+\alpha_{0}\right)}{1-6 \alpha_{0}} E|\zeta-\xi|^{2} \\
& +\frac{6\left(\alpha_{1}+\alpha_{2}+\alpha_{3}\right)}{1-6 \alpha_{0}} \int_{t_{0}}^{t} \Upsilon\left(E\left[\sup _{-\tau \leq r \leq s}|Z(r)-Y(r)|^{2}\right]\right) d s .
\end{aligned}
$$

Finally, using Lemma 2.2 we get

$$
E\left[|Z(t)-Y(t)|^{2}\right] \leq \varepsilon
$$

for $t \in[0, T]$. The proof is complete.

\section{Conclusion}

Neutral stochastic functional differential equations (NSFDEs) play a key role in modeling physical, technical, biological and economic dynamic systems such as predicting option pricing and the growth of populations. In general, one cannot obtain the explicit solutions to NSFDEs. The study of properties and behavior of solutions to NSFDEs such as uniqueness, existence and stability require extensive observations. In this article, we have used some useful inequalities such as the Hölder's inequality, Gronwall's inequality, the Burkholder-Davis-Gundy (in short BDG) inequalities, Bihari's inequality and the Picard approximation scheme to obtain the existence and uniqueness of a solution for neutral stochastic functional differential equation in the G-framework. Moreover, the mean square stability is developed for the above-mentioned stochastic differential equations. The G-Brownian motion theory is not based on a particular probability space and generalizes the classical Brownian motion theory in a non-trivial way. The methodology used in this article to estimate the existence, uniqueness and stability of solutions for NSFDE in the G-framework is attractive and applicable in numerous practical applications. For example, the above-mentioned theory is useful in distributed system control [32], economics [33] and biological as well as neural control systems [34]. This article will play a key role in providing a framework for future work in this direction such as the study of the p-moment estimates for NSFDEs driven by G-Brownian motion and existence theory for stochastic pantograph differential equations driven by G-Brownian motion.

\section{Acknowledgements}

The financial support of TWAS-UNESCO Associateship-Ref. 3240290714 at Centro de Investigación en Matemáticas, A.C. (CIMAT) Jalisco S/N Valenciana A.P. 40236000 Guanajuato, GTO Mexico, is deeply appreciated and acknowledged. We are grateful to the NUST research directorate for providing publication fee and awards. The authors acknowledge and deeply appreciate the careful reading and useful suggestions of the anonymous reviewer, which has improved the quality of this paper.

Competing interests

The authors declare that they have no competing interests.

\section{Authors' contributions}

Subject to non-linear and non-Lipschitz conditions, we have developed the existence theory for the solutions to neutral stochastic functional differential equations (NSDEs) in the framework of G-Brownian motion. By virtue of the Hölder, Gronwall, BDG and Bihari inequalities, we have shown that these equations admit at most one solution. In addition, the mean square stability is established for NSFDEs in the framework of G-Brownian motion. All authors read and approved the final manuscript. 


\section{Author details}

'Department of Basic Sciences and Humanities, College of Electrical and Mechanical Engineering, National University of Sciences and Technology (NUST), Islamabad, Pakistan. ${ }^{2}$ Department of Mathematics and Statistics, Riphah International University, Islamabad, Pakistan. ${ }^{3}$ Department of Mathematics and Statistics, University of Swat, Khyber Pakhtunkhwa, Pakistan.

\section{Publisher's Note}

Springer Nature remains neutral with regard to jurisdictional claims in published maps and institutional affiliations.

\section{Received: 12 May 2017 Accepted: 13 October 2017 Published online: 30 October 2017}

\section{References}

1. Dunzlaff, P, Strauss, RD, Potgieter, MS: Solving Parker's transport equation with stochastic differential equations on GPUs. Comput. Phys. Commun. 192, 156-165 (2015)

2. Zamana, G, Kang, YH, Jung, IH: Stability analysis and optimal vaccination of an SIR epidemic model. Biosystems 93 , 240-249 (2008)

3. Zaman, G, Kang, YH, Cho, G, Jung, IH: Optimal strategy of vaccination \& treatment in an SIR epidemic model. Math. Comput. Simul. 136, 63-77 (2017)

4. Khan, I, Ali, F, Shah, NA: Interaction of magnetic field with heat and mass transfer in free convection flow of a Walters'-B fluid. Eur. Phys. J. Plus 131(77), 1-15 (2016)

5. Gubner, JH: Probability and Random Processes for Electrical and Computer Engineering. Cambridge University Press, Cambridge (2006)

6. Primak, S, Kontorovitch, V, Lyandres, V: Stochastic Methods and Their Applications to Communications: Stochastic Differential Equations Approach. John Wiley \& Sons Ltd, Chichester England (2004)

7. Bolotin, W: Random Vibrations of Elastic Systems. Martinus Nijhoff, The Hague (1984)

8. Roberts, JB, Spanos, PD: Random Vibration and Statistical Linearization. Dover, New York (2003)

9. Skalmierski, B, Tylikowski, A: Stochastic Processes in Dynamics. Polish Scientific Editors, Warsaw (1982)

10. Sobezyk, K: Stochastic Differential Equations with Applications to Physics and Engineering. Kluwer Academic, Dordrecht (1991)

11. Sobezyk, K, Spencer, BF Jr.: Random Fatigue: From Data to Theory. Academic Press, Boston (1992)

12. Skalmierski, B, Tylikowski, A: Stability of Dynamical Systems. Polish Scientific Editors, Warsaw (1973)

13. Sobezyk, K: Stochastic Waive Propagation, Polish Scientific Editors, Warsaw (1984)

14. Peng, S: G-expectation, G-Brownian motion and related stochastic calculus of Ito's type. In: Benth, FE, et al.. (eds.) The Abel Symposium 2005, Abel Symposia 2, pp. 541-567. Springer, Berlin (2006)

15. Peng, S: Multi-dimensional G-Brownian motion and related stochastic calculus under G-expectation. In: Stochastic Processes and Thier Applications, vol. 12, pp. 2223-2253 (2008)

16. Gao, F: Pathwise properties and homeomorphic flows for stochastic differential equations driven by G-Brownian motion. In: Stochastic Processes and Thier Applications, vol. 2, pp. 3356-3382 (2009)

17. Faizullah, F: Existence of solutions for stochastic differential equations under G-Brownian motion with discontinuous coefficients. Z. Naturforsch. A 67A, 692-698 (2012)

18. Gu, Y, Ren, Y, Sakthivel, R: Square-mean pseudo almost automorphic mild solutions for stochastic evolution equations driven by G-Brownian motion. Stoch. Anal. Appl. 34, 528-545 (2016)

19. Ren, Y, Jia, $X$, Sakthivel, R: The $p$ th moment stability of solutions to impulsive stochastic differential equations driven by G-Brownian motion. Appl. Anal. 96, 988-1003 (2017)

20. Ren, Y, Bi, Q, Sakthivel, R: Stochastic functional differential equations with infinite delay driven by G-Brownian motion Math. Methods Appl. Sci. 36(13), 1746-1759 (2013)

21. Faizullah, F: Existence and uniqueness of solutions to SFDEs driven by G-Brownian motion with non-Lipschitz conditions. J. Comput. Anal. Appl. 2(23), 344-354 (2017)

22. Faizullah, F: Existence of solutions for G-SFDEs with Cauchy-Maruyama approximation scheme. Abstr. Appl. Anal. 2014, Article ID 809431 (2014). doi:10.1155/2014/809431, 8 p.

23. Faizullah, F: A note on $p$ th moment estimates for stochastic functional differential equations in the framework of G-Brownian motion. Iran. J. Sci. Technol., Trans. A, Sci. 3(40), 1-8 (2016)

24. Faizullah, F: On the $p$ th moment estimates of solutions to stochastic functional differential equations in the G-framework. SpringerPlus 5(872), 1-11 (2016)

25. Faizullah, F: Existence results and moment estimates for NSFDEs driven by G-Brownian motion. J. Comput. Theor. Nanosci. 7(13), 1-8 (2016)

26. Mao, X: Stochastic Differential Equations and Their Applications. Horwood Publishing, Chichester (1997)

27. Wei, F, Cai, Y: Existence, uniqueness and stability of the solution to neutral stochastic functional differential equations with infinite delay under non-Lipschitz conditions. Adv. Differ. Equ. 2013, 151 (2013)

28. Denis, L, Hu, M, Peng, S: Function spaces and capacity related to a sublinear expectation: application to G-Brownian motion paths. Potential Anal. 34, 139-161 (2010)

29. Hu, M, Peng, S: Extended conditional G-expectations and related stopping times (16 Sep 2013). arXiv:1309.3829v [math.PR]

30. Li, X, Peng, S: Stopping times and related Ito's calculus with G-Brownian motion. In: Stochastic Processes and Thier Applications, vol. 121, pp. 1492-1508 (2011)

31. Song, Y: Properties of hitting times for G-martingale and their applications. In: Stochastic Processes and Thie Applications, vol. 8, pp. 1770-1784 (2011)

32. Shang, Y: Group consensus of multi-agent systems in directed networks with noises and time delays. Int. J. Syst. Sci. 14(46), 2481-2492 (2013)

33. Chow, CH: Optimum control of stochastic differential equation systems. J. Econ. Dyn. Control 1, 143-175 (1979)

34. Sanger, TD: Distributed control of uncertain systems using superpositions of linear operators. Neural Comput. 23(8), 1911-1934 (2011) 